\section{Morbus Bechterew: Studienanalyse findet Prädiktoren für eine erfolgreiche Therapie}

\begin{abstract}
Nicht alle Patienten mit ankylosierender Spondylitis (AS; Morbus Bechterew) sprechen auf eine Behandlung mit nicht steroidalen Antirheumatika (NSAR) an. Welche Parameter sich eignen, um die Erfolgschancen von Biologika vorherzusagen, haben Wissenschaftler aus den USA und Europa untersucht.
\end{abstract}

D as Autorenteam um N. Vastesaeger hat in einer Post-hoc-Analyse die Daten von Patienten ausgewertet, die an der ASSERT- und der GO-RAISE-Studie teilgenommen hatten. Die 635 Patienten (Durchschnittsalter 39,5 Jahre) waren an einer aktiven AS erkrankt. NSAR oder übliche krankheitsmodifizierende Substanzen (Disease Modifying Antirheumatic Drugs, DMARD) hatten nicht ausreichend gewirkt.

In den beiden Studien wurden die ASPatienten einer zusätzlichen Therapie mit den TNF- $\alpha$-Hemmern Infliximab bzw. Golimumab oder Placebo zugeteilt. Für ihre Auswertung analysierten die Autoren diverse klinische oder laborchemische Parameter, deren Werte Hinweise auf die Effizienz der Therapie lieferten. Als Maß für das Ansprechen der Behandlung wurden eine mindestens 50\%ige Verbesserung des Bath Ankylosing Spondylitis Disease Activity Index (BASDAI 50) sowie eine Verbesserung im Assessment of Spondyloarthritis um mindestens 20\% (ASAS 20) gewählt.

In der multivariaten Regressionsanalyse konnten zu Studienbeginn folgende Prädiktoren für eine erfolgreiche $\mathrm{Be}$ handlung identifiziert werden: Alter $<40$ Jahre, niedrige Scorewerte im Bath Ankylosing Spondylitis Functional Index (BASFI), 0 Punkte im Berliner Enthesitis-Score, Behandlung mit TNF- $\alpha$ Hemmern, hohe Konzentrationen des C-reaktiven Proteins (CRP) und positiver HLA-B27-Status.

Die höchsten Werte ergaben sich für die Behandlung mit TNF- $\alpha$-Inhibitoren (Odds-Ratio [OR] bis zu 46,5) und für hohe CRP-Konzentrationen (OR bis zu $15,0)$.

Schlussfolgerung: In der vorliegenden Analyse identifizierten die Autoren eine Reihe von Prädiktoren für die erfolgreiche Behandlung von AS-Patienten. Vor allem hohe CRP-Werte und der Einsatz von TNF- $\alpha$-Hemmern waren mit einem guten Ansprechen der Therapie assoziiert.

Dr. Andreas Fischer

Vastesaeger $\mathrm{N}$ et al. Predicting the outcome of ankylosing spondylitis therapy. Ann Rheum Dis 2011;70:973-81.

Kommentar: Die in dieser Arbeit identifizierten Prädiktoren für ein gutes Ansprechen auf eine Anti-TNF-Therapie bei ASPatienten mit aktiver Erkrankung sind im Wesentlichen bereits bekannt. $\mathrm{Zu}$ ihnen gehören jüngeres Alter (oder kürzere Kankheitsdauer), erhöhtes CRP als Marker der objektiven Entzündung, ein niedriger BASFI (besserer Funktionsstatus) und positiver HLA-B27-Status. Sämtliche Faktoren waren bereits in einer früheren Arbeit identifziert worden (BASFI und HLA-B27 als Trend) [Rudwaleit $M$ et al. Prediction of a major clinical response (BASDAI 50) to tumor necrosis factor alpha blockers in ankylosing spondylitis. Ann Rheum Dis. 2004;63(6):66570] und danach in einer größeren offenen Studie mit 1.250 Patienten [Rudwaleit $M$ et al. Effectiveness, safety, and predictors of good clinical response in 1250 patients treated with adalimumab for active ankylosing spondylitis. J Rheumatol. 2009;36(4): 801-8] und in anderen Studien bestätigt worden.

Neu in dieser Arbeit sind die Hinzunahme des 2009 publizierten ASDAS als OutcomeInstrument vor allem in klinischen Studien und der Enthesitis-Score als Prädiktor (Patienten ohne Enthesitis haben eine etwas größere Chance auf ein gutes Ansprechen als solche mit Enthesitis). Dieses Ergebnis überrascht auf den ersten Blick, da eigentlich auch die Enthesitis unter Anti-TNFTherapie deutlich besser wird [Rudwaleit $M$ et al. Effectiveness of adalimumab in treating patients with ankylosing spondylitis associated with enthesitis and peripheral arthritis. Arthritis Res Ther. 2010;12(2):R43]. Eine mögliche Erklärung hierfür könnte eine insgesamt höhere Krankheitslast bei
Patienten mit Enthesitis sein. Der Effekt der Enthesitis auf die Wahrscheinlichkeit des Ansprechens war zwar signfikant, jedoch nicht sehr groß.

Die Therapie (Anti-TNF) selbst als möglichen Prädiktor für ein gutes Ansprechen auf eine Therapie (entweder mit Placebo oder Anti-TNF) in die Analyse zu nehmen, mag statistisch in der Studienpopulation erlaubt sein, klinisch gesehen ist es jedoch sinnlos. Dass die Anti-TNF-Therapie die höchste Odds Ratio für ein gutes Outcome in dieser Arbeit erhält, ist daher eine logische Konsequenz aus dem Vergleich von Anti-TNF mit Placebo.

In der täglichen Praxis möchte man hingegen wissen, welche Faktoren sich als Prädiktoren für ein gutes Ansprechen bei einem AS-Patienten eignen, für den jetzt eine teure Anti-TNF-Therapie zur Diskussion steht, da der Kranke mit NSAR allein nicht zurecht kommt. Insofern sind auch die Matrix-Tabellen mit den Wahrscheinlichkeiten des Ansprechens von begrenztem Wert. Leider sind sie in der Originalarbeit auch sehr klein und kaum lesbar.

Für die Praxis bleibt festzuhalten, dass AS-Patienten mit aktiver Entzündung und kurzer Krankheitsdauer mit hoher Wahrscheinlichkeit gut auf eine Anti-TNF-Therapie ansprechen. Als Surrogatparameter für eine aktive Entzündung funktioniert im klinischen Alltag auch geringes Alter des Patienten recht gut, denn bei jüngeren AS-Kranken sind die Schmerzen viel häufiger als bei älteren auf AS-Aktivität und nicht auf zusätzliche mechanische/degenerative Veränderungen zurückzuführen.

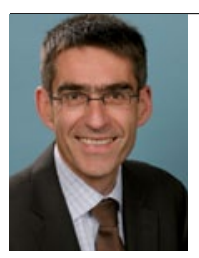

Prof. Dr. med. Martin Rudwaleit Arzt für Innere Medizin, Rheumatologie, Nephrologie, Endokrinologikum Berlin am Gendarmenmarkt Jägerstraße 61 (Q207), 10117 Berlin 